\title{
BOUNDARIES OF LEVI-FLAT HYPERSURFACES: SPECIAL HYPERBOLIC POINTS
}

\author{
PIERRE DOLBEAULT
}

\begin{abstract}
Let $S \subset \mathbb{C}^{n}, n \geq 3$ be a compact connected 2-codimensional submanifold having the following property: there exists a Levi-flat hypersurface whose boundary is $S$, possibly as a current. Our goal is to get examples of such $S$ containing at least one special 1-hyperbolic point: sphere with two horns; elementary models and their gluing. The particular cases of graphs are also described.
\end{abstract}

\section{INTRODUCTION}

Let $S \subset \mathbb{C}^{n}$, be a compact connected 2-codimensional submanifold having the following property: there exists a Levi-flat hypersurface $M \subset \mathbb{C}^{n} \backslash$ $S$ such that $d M=S$ (i.e. whose boundary is $S$, possibly as a current). The case $n=2$ has been intensively studied since the beginning of the eighties, in particular by Bedford, Gaveau, Klingenberg; Shcherbina, Chirka, G. Tomassini, Slodkowski, Gromov, Eliashberg; it needs global conditions: $S$ has to be contained in the boundary of a srictly pseudoconvex domain.

We consider the case $n \geq 3$; results on this case has been obtained since 2005 by Dolbeault, Tomassini and Zaitsev, local necessary conditions recalled in section 2 have to be satisfied by $S$, the singular CR points on $S$ are supposed to be elliptic and the solution $M$ is obtained in the sense of currents [DTZ05, DTZ10]. More recently a regular solution $M$ has been obtained when $S$ satisfies a supplementary global condition as in the case $n=2$ [DTZ09], the singular CR points on $S$ still supposed to be elliptic.

The problem we are interested in is to get examples of such $S$ containing at least one special 1-hyperbolic point (section 2.4). The CR-orbits near a special 1-hyperbolic point are large and, assuming them compact, a careful examination has to be done (sections 2.6, 2.7). As a topological preliminary, we need a generalization of a theorem of Bishop on the difference of the numbers of special elliptic and 1-hyperbolic points (section 2.8); this result is a particular case of a theorem of Hon-Fei Lai [Lai72].

The first considered example is the sphere with two horns which has one special 1-hyperbolic point and three special elliptic points (section 3.4). Then we consider elementary models and their gluing to obtain more complicated examples (section 3.5). Results have been announced in [Dol08, and

Date: November 13, 2018. 
in more precise way in Dol11; the first aim of this paper is to give complete proofs. Finally, we recall in detail and extend the results of [DTZ09] on regularity of the solution when $S$ is a graph satisfying a supplementary global condition, as in the case $n=2$, to the case of existence of special 1-hyperbolic points, and to gluing of elemetary smooth models (section 4).

\section{Preliminaries: LOCAL AND GLOBAL PROPERTIES OF THE BOUNDARY}

2.1. Definitions. A smooth, connected, CR submanifold $M \subset \mathbb{C}^{n}$ is called minimal at a point $p$ if there does not exist a submanifold $N$ of $M$ of lower dimension through $p$ such that $H N=\left.H M\right|_{N}$. By a theorem of Sussman, all possible submanifolds $N$ such that $H N=\left.H M\right|_{N}$ contain, as germs at $p$, one of the minimal possible dimension, defining a so called CR orbit of $p$ in $M$ whose germ at $p$ is uniquely determined.

Let $S$ be a smooth compact connected oriented submanifold of dimension $2 n-2 . \quad S$ is said to be a locally flat boundary at a point $p$ if it locally bounds a Levi-flat hypersurface near $p$. Assume that $S$ is CR in a small enough neighborhood $U$ of $p \in S$. If all CR orbits of $S$ are 1-codimensional (which will appear as a necessary condition for our problem), the following two conditions are equivalent DTZ05]:

(i) $S$ is a locally flat boundary on $U$;

(ii) $S$ is nowhere minimal on $U$.

2.2. Complex points of $S$. (i.e. singular CR points on $S$ ) [DTZ05].

At such a point $p \in S, T_{p} S$ is a complex hyperplane in $T_{p} \mathbb{C}^{n}$. In suitable local holomorphic coordinates $(z, w) \in \mathbb{C}^{n-1} \times \mathbb{C}$ vanishing at $p$, with $w=z_{n}$ and $z=\left(z_{1}, \ldots, z_{n-1}\right), S$ is locally given by the equation

$$
w=Q(z)+O\left(|z|^{3}\right), \quad Q(z)=\sum_{1 \leq i, j \leq n-1}\left(a_{i j} z_{i} z_{j}+b_{i j} z_{i} \bar{z}_{j}+c_{i j} \bar{z}_{i} \bar{z}_{j}\right)
$$

$S$ is said flat at a complex point $p \in S$ if $\sum b_{i j} z_{i} \bar{z}_{j} \in \lambda \mathbf{R}, \lambda \in \mathbb{C}$. We also say that $p$ is flat.

Let $S \subset \mathbb{C}^{n}$ be a locally flat boundary with a complex point $p$. Then $p$ is flat.

By making the change of coordinates $(z, w) \mapsto\left(z, \lambda^{-1} w\right)$, we get $\sum b_{i j} z_{i} z_{j} \in$ $\mathbb{R}$ for all $z$. By a change of coordinates $(z, w) \mapsto\left(z, w+\sum a_{i j}^{\prime} z_{i} z_{j}\right)$ we can choose the holomorphic term in (1) to be the conjugate of the antiholomorphic one and so make the whole form $Q$ real-valued.

We say that $S$ is in a flat normal form at $p$ if the coordinates $(z, w)$ as in (1) are chosen such that $Q(z) \in \mathbf{R}$ for all $z \in \mathbb{C}^{n-1}$.

2.2.1. Properties of $Q$. Assume that $S$ is in a flat normal form; then, the quadratic form $Q$ is real valued. If $Q$ is positive definite or negative definite, the point $p \in S$ is said to be elliptic; if the point $p \in S$ is not elliptic, and if $Q$ is non degenerate, $p$ is said to be hyperbolic. From section 2.4, we will only consider particular cases of the quadratic form $Q$. 


\subsection{Elliptic points.}

\subsubsection{Properties of $Q$.}

Proposition 1. (DTZ05, DTZ10). Assume that $S \subset \mathbb{C}^{n},(n \geq 3)$ is nowhere minimal at all its $C R$ points and has an elliptic flat complex point $p$. Then there exists a neighborhood $V$ of $p$ such that $V \backslash\{p\}$ is foliated by compact real $(2 n-3)$-dimensional $C R$ orbits diffeomorphic to the sphere $\mathbf{S}^{2 n-3}$ and there exists a smooth function $\nu$, having the CR orbits as the level surfaces.

Sketch of Proof. (see [DTZ10]). In the case of a quadric $S_{0}(w=Q(z))$, the $\mathrm{CR}$ orbits are defined by $w_{0}=Q(z)$, where $w_{0}$ is constant. Using (1), we approximate the tangent space to $S$ by the tangent space to $S_{0}$ at a point with the same coordinate $z$; the same is done for the tangent spaces to the CR orbits on $S$ and $S_{0}$; then we construct the global CR orbit on $S$ through any given point close enough to $p$.

2.4. Special flat complex points. From Bis65, for $n=2$, in suitable local holomorphic coordinates centered at $0, \quad Q(z)=\left(z \bar{z}+\lambda \operatorname{Re} z^{2}\right), \quad \lambda \geq 0$, under the notations of [BK91]; for $0 \leq \lambda<1, p$ is said to be elliptic, and for $1<\lambda$, it is said to be hyperbolic. The parabolic case $\lambda=1$, not generic, will be omitted BK91. When $n \geq 3$, the Bishop's reduction cannot be generalized.

We say that the flat complex point $p \in S$ is special if in convenient holomorphic coordinates centered at 0 ,

$$
Q(z)=\sum_{j=1}^{n-1}\left(z_{j} \bar{z}_{j}+\lambda_{j} \operatorname{Re} z_{j}^{2}\right), \quad, \lambda_{j} \geq 0
$$

Let $z_{j}=x_{j}+i y_{j}, x_{j}, y_{j}$ real, $j=1, \ldots, n-1$, then:

$$
Q(z)=\sum_{l=1}^{n-1}\left(\left(1+\lambda_{l}\right) x_{l}^{2}+\left(1-\lambda_{l}\right) y_{l}^{2}\right)+O\left(|z|^{3}\right)
$$

A flat point $p \in S$ is said to be special elliptic if $0 \leq \lambda_{j}<1$ for any $j$.

A flat point $p \in S$ is said to be special k-hyperbolic if $1<\lambda_{j}$ for $j \in J \subset$ $\{1, \ldots, n-1\}$ and $0 \leq \lambda_{j}<1$ for $j \in\{1, \ldots, n-1\} \backslash J \neq \emptyset$, where $k$ denotes the number of elements of $J$.

Special elliptic (resp. special $k$-hyperbolic) points are elliptic (resp. hyperbolic).

Special flat complex points

2.5. Special hyperbolic points. $S$ being given by (1), let $S_{0}$ be the quadric of equation $w=Q(z)$.

Lemma 2. Suppose that $S_{0}$ is flat at 0 and that 0 is a special $k$-hyperbolic point. Then, in a neighborhood of 0 , and with the above local coordinates, $S_{0}$ is $C R$ and nowhere minimal outside 0 , and the $C R$ orbits of $S_{0}$ are the $(2 n-3)$-dimensional submanifolds given by $w=$ const. $\neq 0$. 
Proof. The submanifolds $w=$ const. $\neq 0$ have the same complex tangent space as $S_{0}$ and are of minimal dimension among submanifolds having this property, so they are CR orbits of codimension 1, and from the end of section 2.1. $S_{0}$ is nowhere minimal outside 0 .

The section $w=0$ of $S_{0}$ is a real quadratic cone $\Sigma_{0}^{\prime}$ in $\mathbf{R}^{2 n}$ whose vertex is 0 and, outside 0 , it is a $\mathrm{CR}$ orbit $\Sigma_{0}$ in the neighborhood of 0 . We will improperly call $\Sigma_{0}^{\prime}$ a singular $C R$ orbit.

2.6. Foliation by CR-orbits in the neighborhood of a special 1hyperbolic point. We first mimic and transpose the begining of the proof of Proposition 1, i.e. of 2.4.2. in ([DTZ05, DTZ09]).

2.6.1. Local 2-codimensional submanifolds. In order to use simple notations, we will assume $n=3$.

In $\mathbb{C}^{3}$, consider the 4-dimensional submanifold $S$ locally defined by the equation

$$
w=\varphi(z)=Q(z)+O\left(|z|^{3}\right)
$$

and the 4-dimensional submanifold $S_{0}$ of equation

$$
w=Q(z)
$$

with

$$
Q=\left(\lambda_{1}+1\right) x_{1}^{2}-\left(\lambda_{1}-1\right) y_{1}^{2}+\left(1+\lambda_{2}\right) x_{2}^{2}+\left(1-\lambda_{2}\right) y_{2}^{2}
$$

having a special 1-hyperbolic point at $0,\left(\lambda_{1}>1,0 \leq \lambda_{2}<1\right)$, and the cone $\Sigma_{0}^{\prime}$ whose equation is: $Q=0$. On $S_{0}$, a CR orbit is the 3 -dimensional submanifold $\mathcal{K}_{w_{0}}$ whose equation is $w_{0}=Q(z)$. If $w_{0}>0, \mathcal{K}_{w_{0}}$ does not cut the line $L=\left\{x_{1}=x_{2}=y_{2}=0\right\}$; if $w_{0}<0, \mathcal{K}_{w_{0}}$ cuts $L$ at two points.

Lemma 3. $\Sigma_{0}=\Sigma_{0}^{\prime} \backslash 0$ has two connected components in a neighborhood of 0 .

Proof. The equation of $\Sigma_{0}^{\prime} \cap\left\{y_{1}=0\right\}$ is

$\left(\lambda_{1}+1\right) x_{1}^{2}+\left(1+\lambda_{2}\right) x_{2}^{2}+\left(1-\lambda_{2}\right) y_{2}^{2}=0 \quad$ whose only zero, in the neighborhood of 0 , is $\{0\}$ : the connected components are obtained for $y_{1}>0$ and $y_{1}<0$ respectively.

Local 2-codimensional submanifolds

2.6.2. CR-orbits. By differentiating (1), we get for the tangent spaces the following asymptotics

$$
\left.\left.T_{(z, \varphi(z)}\right) S=T_{(z, Q(z)}\right) S_{0}+O\left(|z|^{2}\right), \quad z \in \mathbb{C}^{2}
$$

Here both $\left.T_{(z, \varphi(z)}\right) S$ and $\left.T_{(z, Q(z)}\right) S_{0}$ depend continuously on $z$ near the origin.

Consider 
(i) the hyperboloï $H_{-}=\{Q=-1\}$, (then $\left.Q\left(\frac{z}{(-Q(z))^{1 / 2}}\right)=-1\right)$, and the projection:

$$
\pi_{-}: \mathbb{C}^{3} \backslash\{z=0\} \rightarrow H_{-}, \quad(z, w) \mapsto \frac{z}{(-Q(z))^{1 / 2}},
$$

(ii) for every $z \in H_{-}$, a real orthonormal basis $e_{1}(z), \ldots, e_{6}(z)$ of $\mathbb{C}^{3} \cong \mathbb{R}^{6}$ such that

$$
e_{1}(z), e_{2}(z) \in H_{z} H_{-}, \quad e_{3}(z) \in T_{z} H_{-},
$$

where $H_{-}$is the complex tangent bundle to $H_{-}$.

Locally such a basis can be chosen continuously depending on $z$. For every $(z, w) \in \mathbb{C}^{3} \backslash\{z=0\}$, consider the basis $e_{1}\left(\pi_{-}(z, w)\right), \ldots, e_{6}\left(\pi_{-}(z, w)\right)$. The unit vectors $e_{1}\left(\pi_{-}\left(z, w_{0}\right)\right), e_{2}\left(\pi_{-}\left(z, w_{0}\right)\right), e_{3}\left(\pi_{-}\left(z, w_{0}\right)\right)$ are tangent to the $\mathrm{CR}$ orbit $\mathcal{K}_{w_{0}}$ in $\left(z, w_{0}\right)$ for $w_{0}<0$. Then, from (5), we have:

$$
\left.\left.H_{(z, \varphi(z)}\right) S=H_{(z, Q(z)}\right) S_{0}+O\left(|z|^{2}\right), \quad z \neq 0, \quad z \rightarrow 0 .
$$

As in [DTZ10], in the neighborhood of 0, denote by $E(q), q \in S \backslash\{0\}, w<0$ the tangent space to the local CR orbit $\mathcal{K}$ on $S$ through $q$, and by $E_{0}\left(q_{0}\right), q_{0} \in$ $S_{0} \backslash\{0\}, w<0$ the analogous object for $S_{0}$. We have :

$$
E(z, \varphi(z))=E_{0}(z, Q(z))+O\left(|z|^{2}\right), \quad z \neq 0, \quad z \rightarrow 0
$$

Given $q \in S$, by integration of $E(q), q \in S$, we get, locally, the CR orbit (the leaf), on $S$ through $\underline{q}$; given $\underline{q}_{0} \in S_{0}$, by integration of $E_{0}\left(q_{0}\right), q_{0} \in S_{0}$, we get, locally, the CR orbit (the leaf), on $S_{0}$ through $\underline{q}_{0}$ (theorem of Sussman). On $S_{0}$, a leaf is the 3 -dimensional submanifold $\mathcal{K}_{q_{0}}=\mathcal{K}_{w_{0}}=\mathcal{K}_{0}$ whose equation is $w_{0}=Q(z)$, with $\underline{q}=\left(z_{0}, w_{0}=Q\left(z_{0}\right)\right)$. $d \pi_{-}$projects each $E_{0}(q), q \in S_{0}, w<0$, bijectively onto $T_{\pi(q)} H_{-}$, then $\pi_{-} \mid \mathcal{K}_{0}$ is a diffeomorphism onto $H_{-}$; this implies, from (7), that, in a suitable neighborhood of the origin, the restriction of $\pi_{-}$to each local CR orbit of $S$ is a local diffeomorphism.

We have: $\varphi(z)=Q(z)+\Phi(z)$ with $\Phi(z)=O\left(|z|^{3}\right)$.

2.6.3. Behaviour of local $C R$ orbits. Follow the construction of $E(z, \varphi(z))$; compare with $E_{0}(z, Q(z))$. We know the integral manifold, the orbit of $E_{0}(z, Q(z))$; deduce an evaluation of the integral manifold $\mathcal{K}$ of $E(z, \varphi(z))$.

Lemma 4. Under the above hypotheses, the local orbit $\Sigma$ corresponding to $\Sigma_{0}$ has two connected components in the neighborhood of 0.

Proof. Using the real coordinates, as for Lemma 3, $\Sigma^{\prime} \cap\left\{y_{1}=0\right\}$. Locally, the connected components are obtained for $y_{1}>0$ and $y_{1}<0$ respectively, from formula (1).

We will improperly call $\Sigma^{\prime}=\bar{\Sigma}$ a singular $C R$ orbit and a singular leaf of the foliation.

We intend to prove: 1) $\mathcal{K}$ does not cross the singular leaf through 0 ; 
2) the only separatrix is the singular leaf through 0 .

From the orbit $\mathcal{K}_{0}$, construct the differential equation defining it, and using (7), construct the differential equation defining $\mathcal{K}$.

In $\mathbb{C}^{3}$, we use the notations: $x=x_{1}, y=y_{1}, u=x_{2}, v=y_{2}$; it suffices to consider the particular case: $Q=3 x^{2}-y^{2}+u^{2}+v^{2}$. On $S_{0}$, the orbit $\mathcal{K}_{0}$ issued from the point $(c, 0,0,0)$ is defined by: $3 x^{2}-y^{2}-u^{2}+v^{2}=3 c^{2}$, i.e., for $x \geq 0, x=\frac{1}{\sqrt{3}}\left(y^{2}-u^{2}-v^{2}+3 c^{2}\right)^{\frac{1}{2}}=A(y, u, v)$; the local coordinates on the orbit are $(y, u, v) . \mathcal{K}_{0}$ satisfies the differential equation: $d x=d A$. From (9), the orbit $\mathcal{K}$, issued from $(c, 0,0,0)$, satisfies $d x=d A+\Psi$ with $\Psi(y, u, v ; c)=O\left(|z|^{2}\right)$; hence $\Psi=d \Phi$, then $x=A+\Phi$, with $\Phi=O\left(|z|^{3}\right)$. More explicitly, $\mathcal{K}$ is defined by:

$$
x=x_{\mathcal{K}, c}=\frac{1}{\sqrt{3}}\left(y^{2}-u^{2}-v^{2}+3 c^{2}\right)^{\frac{1}{2}}+\Phi(y, u, v ; c), \quad \Phi(y, u, v ; c)=O\left(|z|^{3}\right)
$$

The cone $\Sigma_{0}^{\prime}$ whose equation is: $Q=0$ is a separatrix for the orbits $\mathcal{K}_{0}$. The corresponding object $\Sigma^{\prime}=\{\varphi(z)=0\}$ for $S$ has the singular point 0 and for $x>0, y>0, u>0, v>0$ is defined by the differential equation $d x=d(A+\Phi)$, with $c=0$, i.e. the local equation of $\Sigma^{\prime}$ is

$$
x=x_{\mathcal{K}, 0}=\frac{1}{\sqrt{3}}\left(y^{2}-u^{2}-v^{2}\right)^{\frac{1}{2}}+\Phi(y, u, v ; 0), \quad \Phi(y, u, v ; 0)=O\left(|z|^{3}\right)
$$

For given $(y, u, v), x_{\mathcal{K}, c}-x_{\mathcal{K}, 0}=x_{\mathcal{K}_{0}, c}-x_{\mathcal{K}_{0}, 0}+\Phi(y, u, v ; c)-\Phi(y, u, v ; 0)$. But $x_{\mathcal{K}_{0}, c}-x_{\mathcal{K}_{0}, 0}=O(1)$ and $\Phi(y, u, v ; c)-\Phi(y, u, v ; 0)=O\left(|z|^{3}\right)$.

As a consequence, for $x>0, y>0, u>0, v>0$, locally, $\Sigma^{\prime}$ is a separatrix for the orbits $\mathcal{K}$, and the only one. Same result for $x<0$.

2.6.4. What has been done from the hyperboloïd $H_{-}=\{Q=-1\}$ can be repeated from the hyperboloïd $H_{+}=\{Q=1\}$.

As at the beginning of the section 2.6.2, we consider

(i) the hyperboloï $H_{+}\{Q=1\}$ and the projection:

$$
\pi_{+}: \mathbb{C}^{3} \backslash\{z=0\} \rightarrow H_{+}, \quad(z, w) \mapsto \frac{z}{(Q(z))^{1 / 2}},
$$

(ii) for every $z \in H_{+}$, a real orthonormal basis $e_{1}(z), \ldots, e_{6}(z)$ of $\mathbb{C}^{3} \cong \mathbb{R}^{6}$ such that

$$
e_{1}(z), e_{2}(z) \in H_{z} H_{+}, \quad e_{3}(z) \in T_{z} H_{+},
$$

where $\mathrm{HH}_{+}$is the complex tangent bundle to $H_{+}$.

Lemma 5. Given $\varphi$, there exists $R>0$ such that, in $B(0, R) \cap\{x>0, y>$ $0, u>0, v>0\} \subset \mathbb{C}^{2}$, the $C R$ orbits $\mathcal{K}$ have $\Sigma^{\prime}$ as unique separatrix. 
Proof. When c tends to zero,,$x_{\mathcal{K}, c}-x_{\mathcal{K}, 0}=x_{\mathcal{K}_{0}, c}-x_{\mathcal{K}_{0}, 0}=O(|z|)$, $\Phi(y, u, v ; c)-\Phi(y, u, v ; 0)=O\left(|z|^{3}\right)$. For $\varphi(z)=Q(z)+\Phi(z)$ with $\Phi(z)=$ $O\left(|z|^{3}\right)$ given, in $(9), E(z, \varphi(z))-E_{0}(z, Q(z))=O\left(|z|^{2}\right)$ and $\Phi(y, u, v ; c)-$ $\Phi(y, u, v ; 0)=O\left(|z|^{3}\right)$ are also given. Then there exists $R$ such that, for $|z|<R, x_{\mathcal{K}, c}-x_{\mathcal{K}, 0}>0$.

\subsection{CR-orbits near a subvariety containing a special 1-hyperbolic point.}

2.7.1. In the section 2.7, we will impose conditions on $S$ and give a local property in the neighborhood of a compact $(2 n-3)$-subvariety of $S$.

Assume that $S \subset \mathbb{C}^{n}(n \geq 3)$, is a locally closed $(2 n-2)$-submanifold, nowhere minimal at all its $\mathrm{CR}$ points, which has a unique 1-hyperbolic flat complex point $p$, and such that:

(i) $\Sigma$ being the orbit whose closure $\Sigma^{\prime}$ contains $p$, then $\Sigma^{\prime}$ is compact.

Let $q \in S, q \neq p$; then, in a neighborhood $U$ of $q$ disjoint from $p, S$ is CR, CR-dim $S=n-2, S$ is non minimal and $\Sigma$ is 1-codimensional. To show that the $\mathrm{CR}$ orbits contitute a foliation on $S$ whose separatrix is $\Sigma^{\prime}$ : this is true in $U$ since $\Sigma \cap U$ is a leaf. Moreover, let $U_{0}$ the ball $B(0, R)$ centered in $p=0$ in Lemma 5, if $U \cap U_{0} \neq \emptyset$, the leaves in $U$ glue with the leaves in $U_{0}$ on $U \cap U_{0}$. Since $\Sigma^{\prime}$ is compact, there exists a finite number of points $q_{j} \in \Sigma^{\prime}, j=0,1, \ldots, J$, and open neighborhoods $U_{j}$, as above, such that $\left(U_{j}\right)_{j=0}^{J}$ is an open covering of $\Sigma^{\prime}$. Moreover the leaves on $U_{j}$ glue respectively with the leaves on $U_{k}$ if $U_{j} \cap U_{h} \neq \emptyset$.

2.7 .2 .

Proposition 6. Assume that $S \subset \mathbb{C}^{n}(n \geq 3)$, is a locally closed ( $2 n-$ 2)-submanifold, nowhere minimal at all its $C R$ points, which has a unique special 1-hyperbolic flat complex point $p$, and such that:

(i) $\Sigma$ being the orbit whose closure $\Sigma^{\prime}$ contains $p$, then $\Sigma^{\prime}$ is compact;

(ii) $\Sigma$ has two connected components $\sigma_{1}, \sigma_{2}$, whose closures are homeomorphic to spheres of dimension $2 n-3$.

Then, there exists a neighborhood $V$ of $\Sigma^{\prime}$ such that $V \backslash \Sigma^{\prime}$ is foliated by compact real $(2 n-3)$-dimensional $C R$ orbits whose equation, in a neighborhood of $p$ is (3), and, the $w\left(=x_{n}\right)$-axis being assumed to be vertical, each orbit is diffeomorphic to

the sphere $\mathbf{S}^{2 n-3}$ above $\Sigma^{\prime}$,

the union of two spheres $\mathbf{S}^{2 n-3}$ under $\Sigma^{\prime}$, and there exists a smooth function $\nu$, having the $C R$ orbits as the level surfaces.

Proof. From subsection 2.7.1 and the following remark:

When $x_{n}$ tends to 0 , the orbits tends to $\Sigma^{\prime}$, and because of the geometry of the orbits near $p$, they are diffeomorphic to a sphere above $\Sigma^{\prime}$, and to the union of two spheres under $\Sigma^{\prime}$. The existence of $\nu$ is proved as in Proposition 1, namely, consider a smooth curve $\gamma:[0, \varepsilon) \rightarrow S$ such that 
$\gamma(0)=q$, where $q$ is a point of $\Sigma$ close to $p$, and $\gamma$ is a diffeomorphism onto its image $\Gamma=\gamma([0, \varepsilon))$. Let $\nu=\gamma^{-1}$ on the image of $\gamma$, then, close enough to $q$, every $\mathrm{CR}$ orbit cuts $\Gamma$ at a unique point $q(t), t \in[0, \varepsilon)$. Hence there is a unique extension of $\nu$ from $\gamma([0, \varepsilon))$ to $V \backslash p$ where $V$ is a neighborhood of $\Sigma^{\prime}$ having CR orbits as its level surfaces. $\nu$ being smooth away from $p$, it is smooth on the orbit $\Sigma$ and, if we set $\nu(p)=\nu(q)=0, \nu$ is smooth on a neighborhood of $\Sigma \cup\{p\}=\Sigma^{\prime}$.

2.8. Geometry of the complex points of $S$. The results of section 2.8 are particular cases of theorems of H-F Lai [Lai72], that I learnt from F. Forstneric in July 2011.

In [BK91] E. Bedford \& W. Klingenberg cite the following theorem of E. Bishop [Bis65] [section 4, p.15]: On a 2-sphere embedded in $\mathbb{C}^{2}$, the difference between the numbers of elliptic points and of hyperbolic points is the EulerPoincaré characteristic, i.e. 2. For the proof, Bishop uses a theorem of ([CS 51], section 4).

We extend the result for $n \geq 3$ and give proofs which are essentially the same than in the general case of [Lai72, Lai74] but simpler.

2.8.1. Let $S$ be a smooth compact connected oriented submanifold of dimension $2 n-2$. Let $G$ be the manifold of the oriented real linear $(2 n-2)$ subspaces of $\mathbb{C}^{n}$. The submanifold $S$ of $\mathbb{C}^{n}$ has a given orientation which defines an orientation $o(p)$ of the tangent space to $S$ at any point $p \in S$. By mapping each point of $S$ into its oriented tangent space, we get a smooth Gauss map

$$
t: S \rightarrow G
$$

Denote $-t(p)$ the tangent space to $S$ at $p$ with opposite orientation $-o(p)$.

2.8.2. Properties of $G$. (a) $\operatorname{dim} G=2(2 n-2)$.

Proof. $G$ is a two-fold covering of the Grassmannian $M_{m, k}$, of the linear $k$-subspaces of $\mathbb{R}^{m}$ [Ste99] [Part, section 7.9], for $m=2 n$ and $k=2 n-2$; they have the same dimension. We have:

$$
M_{m, k} \cong O_{m} / O_{k} \times O_{m-k}
$$

But $\operatorname{dim} O_{k}=\frac{1}{2} k(k-1)$, hence $\operatorname{dim} M_{m, k}=\frac{1}{2}(m(m-1)-k(k-1)-$ $(m-k)(m-k-1))=k(m-k)$.

(b) $G$ has the complex structure of a smooth quadric of complex dimension $(2 n-2)$ of $\mathbb{C} P^{2 n-1}$ L74, Pol08].

(c) There exists a canonical isomorphism $h: G \rightarrow \mathbb{C} P^{n-1} \times \mathbb{C} P^{n-1}$.

(d) Homology of $G$ (cf Pol08]): Let $S_{1}, S_{2}$ be generators of $H_{2 n-2}(G, \mathbb{Z})$; we assume that $S_{1}$ and $S_{2}$ are fundamental cycles of complex projective subspaces of complex dimension $(n-1)$ of the complex quadric $G$. We also denote $S_{1}, S_{2}$ the ordered two factors $\mathbb{C} P^{n-1}$, so that $h: G \rightarrow S_{1} \times S_{2}$. 


\subsection{3.}

Proposition 7. For $n \geq 2$, in general, $S$ has isolated complex points.

Proof. Let $\pi \in G$ be a complex hyperplane of $\mathbb{C}^{n}$ whose orientation is induced by its complex structure; the set of such $\pi$ is $H=G_{n-1, n}^{\mathbb{C}}=\mathbb{C P}^{n-1 *} \subset$ $G$, as real submanifold. If $p$ is a complex point of $S$, then $t(p) \in H$ or $-t(p) \in H$. The set of complex points of $S$ is the inverse image by $t$ of the intersections $t(S) \cap H$ and $-t(S) \cap H$ in $G$. Since $\operatorname{dim} t(S)=2 n-2$, $\operatorname{dim} H=2(n-1), \operatorname{dim} G=2(2 n-2)$, the intersection is 0-dimensional, in general.

2.8.4. Denoting also $S$, the fundamental cycle of the submanifold $S$ and $t_{*}$ the homomorphism defined by $t$, we have:

$$
t_{*}(S) \sim u_{1} S_{1}+u_{2} S_{2}
$$

where $\sim$ means homologous to.

2.8.5.

Lemma 8 (proved for $n=2$ in [CS51]). With the above notations, we have: $u_{1}=u_{2} ; u_{1}+u_{2}=\chi(S)$, Euler-Poincaré characteristic of $S$.

The proof for $n=2$ works for any $n \geq 3$, namely:

Let $G^{\prime}$ be the manifold of the oriented real linear 2-subspaces of $\mathbb{C}^{n}$. Let $\alpha: G \rightarrow G^{\prime}$ map each oriented $2(n-1)$-subspace $R$ onto its normal 2subspace $R^{\prime}$ oriented so that $R, R^{\prime}$ determine the orientation of $\mathbb{C}^{n} . \quad \alpha$ is a canonical isomorphism. Let $n: S \rightarrow G^{\prime}$ the map defined by taking oriented normal planes; then: $n=\alpha t$ and $t=\alpha^{-1} n$, hence the mapping $h \alpha h^{-1}$ : $S_{1} \times S_{2} \rightarrow S_{1} \times S_{2}$. Let $(x, y)$ be a point of $S_{1} \times S_{2}$, then (†) $h \alpha h^{-1}(x, y)=$ $(x,-y)$.

Over $G$, there is a bundle $V$ of spheres obtained by considering as fiber over a real oriented linear $(2 n-2)$-subspace of $\mathbb{C}^{n}$ through 0 the unit sphere $\mathbf{S}^{2 n-3}$ of this subspace. Let $\Omega$ be the characteristic class of $V$, and let $\Omega_{t}$, $\Omega_{n}$ denote the characteristic classes of the tangent and normal bundles of $S$. Then $t^{*} \Omega=\Omega_{t}, n^{*} \Omega=\Omega_{n}$.

$V$ is the Stiefel manifold of ordered pairs of orthogonal unit vectors through in $\mathbb{R}^{2 n} \cong \mathbb{C}^{n}$. Let $f: V \rightarrow G$ the projection.

From the Gysin sequence, we see that the kernel of $f^{*}: H^{2 n-2}(G) \rightarrow$ $H^{2 n-2}(V)$ is generated by $\Omega$. To find the kernel of $f^{*}$, we determine the morphism $\quad f_{*}: H_{2 n-2}(V) \rightarrow H_{2 n-2}(G)$. A generating $2 n-2$ )-cycle of in $V$ is $S^{2} \times e$ where $S^{2} \cong \mathbb{C} P^{n-1}$ and $e$ is a point. Let $z$ be any point of $S^{2}$, then from $(\dagger)$, we have

$$
h f(z, e)=(z,-z)
$$

Therefore, we see that $f_{*}\left(S^{2} \times e\right)=S_{1}-S_{2}$. Then, the kernel of $f^{*}$ is $\mathbb{Z}$-generated by $S_{1}^{*}+S_{2}^{*}$. 
With convenient orientation for the fibre of the bundle $V$, we get: $\Omega=$ $S_{1}^{*}+S_{2}^{*}$. For convenient orientation of $S$, we get $\Omega_{t} . S=\chi_{S}=$ Euler characteristic of $S$. We have

$$
\begin{gathered}
\Omega_{t}=t^{*}\left(S_{1}^{*}+S_{2}^{*}\right)=t^{*} S_{1}^{*}+t^{*} S_{2}^{*} \\
\Omega_{n}=n^{*}\left(S_{1}^{*}+S_{2}^{*}\right)=t^{*} \alpha^{*}\left(S_{1}^{*}+S_{2}^{*}\right)=t^{*}\left(S_{1}^{*}-S_{2}^{*}\right)=t^{*} S_{1}^{*}-t^{*} S_{2}^{*}
\end{gathered}
$$

Since $\Omega_{n}=0$, we get:

$$
\left(t^{*} S_{1}^{*}\right) . S=\left(t^{*} S_{2}^{*}\right) \cdot S=\frac{1}{2} \chi_{S}
$$

2.8.6. Local intersection numbers of $H$ and $t(S)$ when all complex points are flat and special. $H$ is a complex linear $(n-1)$-subspace of $G$, then is homologous to one of the $S_{j}, j=1,2$, say $S_{2}$ when $G$ has its structure of complex quadric. The intersection number of $H$ and $S_{1}$ is 1 and the intersection number of $H$ and $S_{2}$ is 0 . So, the intersection number of $H$ and $u_{1} S_{1}+u_{2} S_{2}$ is $u_{1}$.

In the neighborhood of a complex point $0, S$ is defined by equation (1), with $w=z_{n}$ and

$$
Q(z)=\sum_{j=1}^{n-1} \mu_{j}\left(z_{j} \bar{z}_{j}+\lambda_{j} \mathcal{R} e z_{j}^{2}\right), \quad \mu_{j}>0, \lambda_{j} \geq 0
$$

Let $z_{j}=x_{2 j-1}+i x_{2 j}, \quad j=1, \ldots, n$, with real $x_{l}$. Let $e_{l}$ the unit vector of the $x_{l}$ axis, $l=1, \ldots, 2 n$.

For simplicity assume $n=3: \quad Q(z)=\mu_{1}\left(z_{1} \bar{z}_{1}+\lambda_{1} \mathcal{R} e z_{1}^{2}\right)+\mu_{2}\left(z_{2} \bar{z}_{2}+\right.$ $\lambda_{2} \mathcal{R}$ e $z_{2}^{2}$ ), with $\mu_{1}=\mu_{2}=1$.

Then, up to higher order terms, $S$ is defined by:

$z_{1}=x_{1}+i x_{2} ; \quad z_{2}=x_{3}+i x_{4} ; \quad z_{3}=\left(1+\lambda_{1}\right) x_{1}^{2}+\left(1-\lambda_{1}\right) x_{2}^{2}+\left(1+\lambda_{2}\right) x_{3}^{2}+$ $\left(1-\lambda_{2}\right) x_{4}^{2}$.

In the neighborhood of 0 , the tangent space to $S$ is defined by the four independent vectors

$$
\begin{array}{r}
\nu_{1}=e_{1}+2\left(1+\lambda_{1}\right) x_{1} e_{5} ; \quad \nu_{2}=e_{2}+2\left(1-\lambda_{1}\right) x_{2} e_{5} ; \quad \nu_{3}=e_{3}+2\left(1+\lambda_{2}\right) x_{3} e_{5} ; \\
\nu_{4}=e_{4}+2\left(1-\lambda_{2}\right) x_{4} e_{5}
\end{array}
$$

Then, if 0 is special elliptic or special $k$-hyperbolic with $k$ even, the tangent plane at 0 has the same orientation; if 0 is special elliptic or special $k$-hyperbolic with $k$ odd the tangent space has opposite orientation.

2.8.7.

Proposition 9 (known for $n=2$ [Bis65], here for $n \geq 3$ ). Let $S$ be a smooth, oriented, compact, 2-codimensional, real submanifold of $\mathbb{C}^{n}$ whose all complex points are flat and special elliptic or special 1-hyperbolic. Then, on $S, \sharp$ (special elliptic points) - $\sharp$ (special 1-hyperbolic points $=\chi(S)$. If $S$ is a sphere, this number is 2. 
Proof. Let $p \in S$ be a complex point and $\pi$ be the tangent hyperplane to $S$ at $\pi$. Assume that

$(* *)$ the orientation of $S$ induces, on $\pi$, the orientation given by its complex structure, then $\pi \in H$.

If $p$ is elliptic, the intersection number of $H$ and $t(S)$ is 1 ; if $p$ is 1hyperbolic, the intersection number of $H$ and $t(S)$ is -1 at $p$.

From the beginning of section 2.8.6, the sum of the intersection numbers of $H$ and $t(S)$ at complex points $p$ satisfying $\left({ }^{*}\right)$ is $u_{1}$. Reversing the condition $(* *)$, and using Lemma 8, we get the Proposition.

\section{Particular CASES: HORNED SPhere; Elementary MOdels AND THEIR GLUING}

3.1. We recall the following Harvey-Lawson theorem with real parameter to be used later.

3.1.1. Let $E \cong \mathbf{R} \times \mathbb{C}^{n-1}$, and $k: \mathbf{R} \times \mathbb{C}^{n-1} \rightarrow \mathbf{R}$ be the projection. Let $N \subset E$ be a compact, (oriented) CR subvariety of $\mathbb{C}^{n+1}$ of real dimension $2 n-2$ and $\mathrm{CR}$ dimension $n-2,(n \geq 3)$, of class $C^{\infty}$, with negligible singularities (i.e. there exists a closed subset $\tau \subset N$ of $(2 n-2)$-dimensional Hausdorff measure 0 such that $N \backslash \tau$ is a CR submanifold). Let $\tau^{\prime}$ be the set of all points $z \in N$ such that either $z \in \tau$ or $z \in N \backslash \tau$ and $N$ is not transversal to the complex hyperplane $k^{-1}(k(z))$ at $z$. Assume that $N$, as a current of integration, is $d$-closed and satisfies:

(H) there exists a closed subset $L \subset \mathbb{R}_{x_{1}}$ with $H^{1}(L)=0$ such that for every $x \in k(N) \backslash L$, the fiber $k^{-1}(x) \cap N$ is connected and does not intersect $\tau^{\prime}$.

\section{1 .2 .}

Theorem 10 ([DTZ10] (see also DTZ05)). Let $N$ satisfy $(\mathrm{H})$ with $L$ chosen accordingly. Then, there exists, in $E^{\prime}=E \backslash k^{-1}(L)$, a unique $C^{\infty}$ Levi-flat $(2 n-1)$-subvariety $M$ with negligible singularities in $E^{\prime} \backslash N$, foliated by complex $(n-1)$-subvarieties, with the properties that $M$ simply (or trivially) extends to $E^{\prime}$ as a $(2 n-1)$-current (still denoted $M$ ) such that $d M=N$ in $E^{\prime} .1$ The leaves are the sections by the hyperplanes $E_{x_{1}^{0}}, x_{1}^{0} \in k(N) \backslash L$, and are the solutions of the "Harvey-Lawson problem" for finding a holomorphic subvariety in $E_{x_{1}^{0}} \cong \mathbb{C}^{n}$ with prescribed boundary $N \cap E_{x_{1}^{0}}$.

\subsection{3.}

Remark 11. Theorem 10 is valid in the space $E \cap\left\{\alpha_{1}<x_{1}<\alpha_{2}\right\}$, with the corresponding condition (H). Moreover, since $N$ is compact, for convenient coordinate $x_{1}$, we can assume $x_{1} \in[0,1]$. 
3.2. To solve the boundary problem by Levi-flat hypersurfaces, $S$ has to satisfy necessary and sufficient local conditions. A way to prove that these conditions can occur is to construct an example for which the solution is obvious.

\subsection{Sphere with one special 1-hyperbolic point (sphere with two horns): Example.}

3.3.1. In $\mathbb{C}^{3}$, let $\left(z_{j}\right), j=1,2,3$, be the complex coordinates and $z_{j}=$ $x_{j}+i y_{j}$. In $\mathbf{R}^{6} \cong \mathbb{C}^{3}$, consider the 4-dimensional subvariety (with negligible singularities) $S$ defined by:

$$
\begin{aligned}
& y_{3}=0 \\
& 0 \leq x_{3} \leq 1 ; \quad x_{3}\left(x_{1}^{2}+y_{1}^{2}+x_{2}^{2}+y_{2}^{2}+x_{3}^{2}-1\right)+\left(1-x_{3}\right)\left(x_{1}^{4}+y_{1}^{4}+x_{2}^{4}+y_{2}^{4}+\right. \\
& \left.4 x_{1}^{2}-2 y_{1}^{2}+x_{2}^{2}+y_{2}^{2}\right)=0 \\
& -1 \leq x_{3} \leq 0 ; \quad x_{3}=x_{1}^{4}+y_{1}^{4}+x_{2}^{4}+y_{2}^{4}+4 x_{1}^{2}-2 y_{1}^{2}+x_{2}^{2}+y_{2}^{2}
\end{aligned}
$$

The singular set of $S$ is the 3 -dimensional section $x_{3}=0$ along which the tangent space is not everywhere (uniquely) defined. $S$ being in the real hyperplane $\left\{y_{3}=0\right\}$, the complex tangent spaces to $S$ are $\left\{x_{3}=x^{0}\right\}$ for convenient $x^{0}$.

3.3.2. The tangent space to the hypersurface $f\left(x_{1}, y_{1}, x_{2}, y_{2}, x_{3}\right)=0$ in $\mathbb{R}^{5}$ is

$$
X_{1} f_{x_{1}}^{\prime}+Y_{1} f_{y_{1}}^{\prime}+X_{2} f_{x_{2}}^{\prime}+Y_{2} f_{y_{2}}^{\prime}+X_{3} f_{x_{3}}^{\prime}=0,
$$

Then, the tangent space to $S$ in the hyperplane $\left\{y_{3}=0\right\}$ is:

for $0 \leq x_{3}$,

$$
\begin{aligned}
& 2 x_{1}\left[x_{3}+2\left(1-x_{3}\right)\left(x_{1}^{2}+2\right)\right] X_{1}+2 y_{1}\left[x_{3}+2\left(1-x_{3}\right)\left(y_{1}^{2}-1\right)\right] Y_{1} \\
& +2 x_{2}\left[x_{3}+\left(1-x_{3}\right)\left(2 x_{2}^{2}+1\right)\right] X_{2}+2 y_{2}\left[x_{3}+\left(1-x_{3}\right)\left(2 y_{2}^{2}+1\right)\right] Y_{2} \\
& \quad+\left[\left(x_{1}^{2}+y_{1}^{2}+x_{2}^{2}+y_{2}^{2}+3 x_{3}^{2}-1\right)\right. \\
& \left.\quad-\left(x_{1}^{4}+y_{1}^{4}+x_{2}^{4}+y_{2}^{4}+4 x_{1}^{2}-2 y_{1}^{2}+x_{2}^{2}+y_{2}^{2}\right)\right] X_{3}=0
\end{aligned}
$$

for $x_{3} \leq 0$,

$4\left(x_{1}^{2}+2\right) x_{1} X_{1}+4\left(y_{1}^{2}-1\right) y_{1} Y_{1}+2\left(2 x_{2}^{2}+1\right) x_{2} X_{2}+2\left(2 y_{2}^{2}+1\right) y_{2} Y_{2}-X_{3}=0$.

3.3.3. The complex points of $S$ are defined by the vanishing of the coefficients of $X_{j}, \mathrm{j}=1,2,3,4$ in the equation of the tangent spaces

for $0 \leq x_{3} \leq 1$,

$$
\begin{aligned}
& x_{1}\left[x_{3}+2\left(1-x_{3}\right)\left(x_{1}^{2}+2\right)\right]=0 \\
& y_{1}\left[x_{3}+2\left(1-x_{3}\right)\left(y_{1}^{2}-1\right)\right]=0 \\
& x_{2}\left[x_{3}+\left(1-x_{3}\right)\left(2 x_{2}^{2}+1\right)\right]=0 \\
& y_{2}\left[x_{3}+\left(1-x_{3}\right)\left(2 y_{2}^{2}+1\right)\right]=0
\end{aligned}
$$

We have the solutions 
$h: x_{j}=0, y_{j}=0,(j=1,2), x_{3}=0$

$e_{3}: x_{j}=0, y_{j}=0,(j=1,2), x_{3}=1$.

for $x_{3} \leq 0$,

$$
\begin{aligned}
& \left(x_{1}^{2}+2\right) x_{1}=0, \\
& \left(y_{1}^{2}-1\right) y_{1}=0 \\
& \left(2 x_{2}^{2}+1\right) x_{2}=0, \\
& \left(2 y_{2}^{2}+1\right) y_{2}=0 .
\end{aligned}
$$

We have the solutions

$h: x_{j}=0, y_{j}=0,(j=1,2), x_{3}=0$;

$e_{1}, e_{2}: x_{1}=0, y_{1}= \pm 1, x_{2}=0, y_{2}=0, x_{3}=-1$.

Remark that the tangent space to $S$ at $h$ is well defined. Moreover, the set $S$ will be smoothed along its section by the hyperplane $\left\{x_{3}=0\right\}$ by a small deformation leaving $h$ unchanged. In the following $S$ will denote this smooth submanifold.

\subsection{4.}

Lemma 12. The points $e_{1}, e_{2}, e_{3}$ are special elliptic; the point $h$ is special $\{1\}$-hyperbolic.

Proof. Point $e_{3}$ : Let $x_{3}^{\prime}=1-x_{3}$, then the equation of $S$ in the neighborhood of $e_{3}$ is:

$\left(1-x_{3}^{\prime}\right)\left(x_{1}^{2}+y_{1}^{2}+x_{2}^{2}+y_{2}^{2}+x_{3}^{\prime 2}-2 x_{3}^{\prime}\right)-x_{3}^{\prime}\left(x_{1}^{4}+y_{1}^{4}+x_{2}^{4}+y_{2}^{4}+4 x_{1}^{2}-\right.$ $\left.2 y_{1}^{2}+x_{2}^{2}+y_{2}^{2}\right)=0$, i.e.

$\left.2 x_{3}^{\prime}=x_{1}^{2}+y_{1}^{2}+x_{2}^{2}+y_{2}^{2}\right)+O\left(|z|^{3}\right)$, or $w=z \bar{z}+O\left(|z|^{3}\right)$

then $e_{3}$ is special elliptic.

Points $e_{1}, e_{2}$ : Let $y_{1}^{\prime}=y_{1} \pm 1, x_{3}^{\prime}=x_{3}+1$, then the equation of $S$ in the neighborhood of $e_{1}, e_{2}$ is:

$$
\begin{aligned}
& x_{3}^{\prime}-1=x_{1}^{4}+\left(y_{1}^{\prime} \mp 1\right)^{4}+x_{2}^{4}+y_{2}^{4}+4 x_{1}^{2}-2\left(y_{1}^{\prime} \mp 1\right)^{2}+x_{2}^{2}+y_{2}^{2} \\
& =x_{1}^{4}+y_{1}^{\prime 4} \mp 4 y_{1}^{\prime 3}+6 y_{1}^{\prime 2} \mp 4 y_{1}^{\prime}+1+x_{2}^{4}+y_{2}^{4}+4 x_{1}^{2}-2\left(y_{1}^{\prime} \mp 1\right)^{2}+x_{2}^{2}+y_{2}^{2},
\end{aligned}
$$
then

$$
\begin{aligned}
& x_{3}^{\prime}=x_{1}^{4}+y_{1}^{\prime} \mp 4 y_{1}^{\prime 3}+4 y_{1}^{\prime 2}+x_{2}^{4}+y_{2}^{4}+4 x_{1}^{2}+x_{2}^{2}+y_{2}^{2}, \text { i.e. } \\
& x_{3}^{\prime}=4 x_{1}^{2}+4 y_{1}^{\prime 2}+x_{2}^{2}+y_{2}^{2}+O\left(|z|^{3}\right), \text { or } w=4 z_{1} \bar{z}_{1}+z_{2} \bar{z}_{2},
\end{aligned}
$$

then $e_{1}, e_{2}$ are special elliptic.

Point $h$ : The equation of $S$ in the neighborhood of $h$ is:

for $x_{3} \geq 0$,

$$
\begin{aligned}
x_{3}\left(x_{1}^{2}+y_{1}^{2}+\right. & \left.x_{2}^{2}+y_{2}^{2}+x_{3}^{2}-1\right) \\
& +\left(1-x_{3}\right)\left(x_{1}^{4}+y_{1}^{4}+x_{2}^{4}+y_{2}^{4}+4 x_{1}^{2}-2 y_{1}^{2}+x_{2}^{2}+y_{2}^{2}\right)=0
\end{aligned}
$$

for $x_{3} \leq 0$,

$$
x_{3}=x_{1}^{4}+y_{1}^{4}+x_{2}^{4}+y_{2}^{4}+4 x_{1}^{2}-2 y_{1}^{2}+x_{2}^{2}+y_{2}^{2} \text {, i.e. }
$$

$x_{3}=4 x_{1}^{2}-2 y_{1}^{2}+x_{2}^{2}+y_{2}^{2}+O\left(|z|^{3}\right)$, in both cases, up to the third order terms, i.e.: $w=z_{1} \bar{z}_{1}+z_{2} \bar{z}_{2}+3 \mathcal{R}$ e $z_{1}^{2}$,

then $h$ is special $\{1\}$-hyperbolic. 
3.3.5. Section $\Sigma^{\prime}=S \cap\left\{x_{3}=0\right\}$. Up to a small smooth deformation, its equation is:

$$
x_{1}^{4}+y_{1}^{4}+x_{2}^{4}+y_{2}^{4}+4 x_{1}^{2}-2 y_{1}^{2}+x_{2}^{2}+y_{2}^{2}=0 \text {, in }\left\{x_{3}=0\right\} .
$$

The tangent cone to $\Sigma^{\prime}$ at 0 is: $4 x_{1}^{2}-2 y_{1}^{2}+x_{2}^{2}+y_{2}^{2}=0$.

Locally, the section of $S$ by the coordinate 3 -space

$$
\begin{array}{ll}
x_{1}, y_{1}, x_{3} \text { is: } & x_{3}=4 x_{1}^{2}-2 y_{1}^{2}+O\left(|z|^{3}\right) \\
x_{2}, y_{2}, x_{3} \text { is: } & x_{3}=x_{2}^{2}+y_{2}^{2}+O\left(|z|^{3}\right)
\end{array}
$$

3.3.1'. Shape of $\Sigma^{\prime}=S \cap\left\{x_{3}=0\right\}$ in the neighborhood of the origin 0 of $\mathbb{C}^{3}$.

Lemma 13. Under the above hypotheses and notations,

(i) $\Sigma=\Sigma^{\prime} \backslash 0$ has two connected components $\sigma_{1}, \sigma_{2}$.

(ii) The closures of the three connected components of $S \backslash \Sigma^{\prime}$ are submanifolds with boundaries and corners.

Proof. ( $i$ ) The only singular point of $\Sigma^{\prime}$ is 0 . We work in the ball $B(0, A)$ of $\mathbb{C}^{2}\left(x_{1}, y_{1}, x_{2}, y_{2}\right)$ for small $A$ and in the 3 -space $\pi_{\lambda}=\left\{y_{2}=\lambda x_{2}\right\}, \lambda \in$ $\mathbb{R}$. For $\lambda$ fixed, $\pi_{\lambda} \cong \mathbb{R}^{3}\left(x_{1}, y_{1}, x_{2}\right)$, and $\Sigma^{\prime} \cap \pi_{\lambda}$ is the cone of equation $4 x_{1}^{2}-2 y_{1}^{2}+\left(1+\lambda^{2}\right) x_{2}^{2}+O\left(|z|^{3}\right)=0$ with vertex 0 and basis in the plane $x_{2}=x_{2}^{0}$ the hyperboloid $H_{\lambda}$ of equation $4 x_{1}^{2}-2 y_{1}^{2}+\left(1+\lambda^{2}\right) x_{2}^{02}+O\left(|z|^{3}\right)=0$; the curves $H_{\lambda}$ have no common point outside 0 . So, when $\lambda$ varies, the surfaces $\Sigma^{\prime} \cap \pi_{\lambda}$ are disjoint outside 0 . The set $\Sigma^{\prime}$ is clearly connected; $\Sigma^{\prime} \cap\left\{y_{1}=0\right\}=\{0\}$, the origin of $\mathbb{C}^{3}$; from above: $\sigma_{1}=\Sigma \cap\left\{y_{1}>0\right\}$; $\sigma_{2}=\Sigma \cap\left\{y_{1}<0\right\}$.

(ii) The three connected components of $S \backslash \Sigma^{\prime}$ are the components which contain, respectively $e_{1}, e_{2}, e_{3}$ and whose boundaries are $\bar{\sigma}_{1}, \bar{\sigma}_{2}, \bar{\sigma}_{1} \cup \bar{\sigma}_{2}$; these boundaries have corners as shown in the first part of the proof.

The connected component of $\mathbb{C}^{2} \times \mathbb{R} \backslash S$ containing the point $(0,0,0,0,1 / 2)$ is the Levi-flat solution, the complex leaves being the sections by the hyperplanes $x_{3}=x_{3}^{0},-1<x_{3}^{0}<1$.

The sections by the hyperplanes $x_{3}=x_{3}^{0}$ are diffeomorphic to a 3 -sphere for $0<x_{3}^{0}<1$ and to the union of two disjoint 3-spheres for $-1<x_{3}^{0}<0$, as can be shown intersecting $S$ by lines through the origin in the hyperplane $x_{3}=x_{3}^{0} ; \Sigma^{\prime}$ is homeomorphic to the union of two 3 -spheres with a common point.

The connected component of $\mathbb{C}^{2} \times \mathbb{R} \backslash S$ containing the point $(0,0,0,0,1 / 2)$ is the Levi-flat solution, the complex leaves being the sections by the hyperplanes $x_{3}=x_{3}^{0},-1<x_{3}^{0}<1$.

The sections by the hyperplanes $x_{3}=x_{3}^{0}$ are diffeomorphic to a 3 -sphere for $0<x_{3}^{0}<1$ and to the union of two disjoint 3 -spheres for $-1<x_{3}^{0}<0$, as can be shown intersecting $S$ by lines through the origin in the hyperplane $x_{3}=x_{3}^{0} ; \Sigma^{\prime}$ is homeomorphic to the union of two 3 -spheres with a common point.

3.4. Sphere with one special 1-hyperbolic point (sphere with two horns). The example of section 3.3 shows that the necessary conditions of 
section 2 can be realised. Moreover, from Proposition 2.8.7, the hypothesis on the number of complex points is meaningful.

\subsection{1.}

Proposition 14. [cf [Dol08][Proposition 2.6.1]] Let $S \subset \mathbb{C}^{n}$ be a compact connected real 2-codimensional manifold such that the following holds:

(i) $S$ is a topological sphere; $S$ is nonminimal at every $C R$ point;

(ii) every complex point of $S$ is flat; there exist three special elliptic points $e_{j}, j=1,2,3$ and one special 1-hyperbolic point $h$;

(iii) $S$ does not contain complex manifolds of dimension $(n-2)$;

(iv) the singular $\mathrm{CR}$ orbit $\Sigma^{\prime}$ through $h$ on $S$ is compact and $\Sigma^{\prime} \backslash\{h\}$ has two connected components $\sigma_{1}$ and $\sigma_{2}$ whose closures are homeomorphic to spheres of dimension $2 n-3$;

(v) the closures $S_{1}, S_{2}, S_{3}$ of the three connected components $S_{1}^{\prime}, S_{2}^{\prime}, S_{3}^{\prime}$ of $S \backslash \Sigma^{\prime}$ are submanifolds with (singular) boundary.

Then each $S_{j} \backslash\left\{e_{j} \cup \Sigma^{\prime}\right\}, j=1,2,3$ carries a foliation $\mathcal{F}_{j}$ of class $C^{\infty}$ with 1-codimensional $\mathrm{CR}$ orbits as compact leaves.

Proof. From conditions (i) and (ii), $S$ satisfying the hypotheses of Proposition 1, near any elliptic flat point $e_{j}$, and of Proposition [6 near $\Sigma^{\prime}$, all CR orbits being diffeomorphic to the sphere $\mathbf{S}^{2 n-3}$. The assumption (iii) guarantees that all CR orbits in $S$ must be of real dimension $2 n-3$. Hence, by removing small connected open saturated neighborhoods of all special elliptic points, and of $\Sigma^{\prime}$, we obtain, from $S \backslash \Sigma^{\prime}$, three compact manifolds $S_{j}{ }^{\prime}, j=1,2,3$, with boundary and with the foliation $\mathcal{F}_{j}$ of codimension 1 given by its $\mathrm{CR}$ orbits whose first cohomology group with values in $\mathbf{R}$ is 0 , near $e_{j}$. It is easy to show that this foliation is transversely oriented.

3.4.2. Recall the Thurston's Stability Theorem ([ $\mathrm{CaC}]$, Theorem 6.2.1).

Proposition 15. Let $(M, \mathcal{F})$ be a compact, connected, transversely-orientable, foliated manifold with boundary or corners, of codimension 1, of class $C^{1}$. If there is a compact leaf $L$ with $H^{1}(L, \mathbf{R})=0$, then every leaf is homeomorphic to $L$ and $M$ is homeomorphic to $L \times[0,1]$, foliated as a product,

Then, from the above theorem, $S_{j}$ " is homeomorphic to $\mathbf{S}^{2 n-3} \times[0,1]$ with CR orbits being of the form $\mathbf{S}^{2 n-3} \times\{x\}$ for $x \in[0,1]$. Then the full manifold $S_{j}$ is homeomorphic to a half-sphere supported by $\mathbf{S}^{2 n-2}$ and $\mathcal{F}_{j}$ extends to $S_{j} ; S_{3}$ having its boundary pinched at the point $h$.

\subsection{3.}

Theorem 16. Let $S \subset \mathbb{C}^{n}, n \geq 3$, be a compact connected smooth real 2codimensional submanifold satisfying the conditions $(i)$ to $(v)$ of Proposition 15. Then there exists a Levi-flat $(2 n-1)$-subvariety $\tilde{M} \subset \mathbb{C} \times \mathbb{C}^{n}$ with boundary $\tilde{S}$ (in the sense of currents) such that the natural projection $\pi$ : 
$\mathbb{C} \times \mathbb{C}^{n} \rightarrow \mathbb{C}^{n}$ restricts to a bijection which is a $C R$ diffeomorphism between $\tilde{S}$ and $S$ outside the complex points of $S$.

Proof. By Proposition 1, for every $e_{j}$, a continuous function $\nu_{j}^{\prime}, C^{\infty}$ outside $e_{j}$, can be constructed in a neighborhood $U_{j}$ of $e_{j}, j=1,2,3$, and by Proposition 6, we have an analogous result in a neighborhood of $\Sigma^{\prime}$. Furthermore, from Proposition 15, a smooth function $\nu^{\prime \prime}{ }_{j}$ whose level sets are the leaves of $\mathcal{F}_{j}$ can be obtained globally on $S_{j}^{\prime} \backslash\left\{e_{j} \cup \Sigma^{\prime}\right\}$. With the functions $\nu_{j}^{\prime}$ and $\nu^{\prime}{ }_{j}$, and analogous functions near $\Sigma^{\prime}$, then using a partition of unity, we obtain a global smooth function $\nu_{j}: S_{j} \rightarrow \mathbf{R}$ without critical points away from the complex points $e_{j}$ and from $\Sigma^{\prime}$.

Let $\sigma_{1}$, resp. $\sigma_{2}$ be the two connected, relatively compact components of $\Sigma \backslash\{h\}$, according to condition $(i v) ; \bar{\sigma}_{1}$, resp. $\bar{\sigma}_{2}$ are the boundary of $S_{1}$, resp. $S_{2}$, and $\bar{\sigma}_{1} \cup \bar{\sigma}_{2}$ the boundary of $S_{3}$. We can assume that the three functions $\nu_{j}$ are finite valued and get the same values on $\bar{\sigma}_{1}$ and $\bar{\sigma}_{2}$. Hence a function $\nu: S \rightarrow \mathbf{R}$.

The submanifold $S$ being, locally, a boundary of a Levi-flat hypersurface, is orientable. We now set $\tilde{S}=N=\operatorname{gr} \nu=\{(\nu(z), z): z \in S\}$. Let $S_{s}=\left\{e_{1}, e_{2}, e_{3}, \overline{\sigma_{1} \cup \sigma_{2}}\right\}$.

$\lambda: S \rightarrow \tilde{S}(z \mapsto \nu((z), z))$ is bicontinuous; $\left.\lambda\right|_{S \backslash S_{s}}$ is a diffeomorphism; moreover $\lambda$ is a CR map. Choose an orientation on $S$. Then $N$ is an (oriented) CR subvariety with the negligible set of singularities $\tau=\lambda\left(S_{s}\right)$.

At every point of $S \backslash S_{s}, d_{x_{1}} \nu \neq 0$, then condition (H) (section 3.1.1) is satisfied at every point of $N \backslash \tau$.

Then all the assumptions of Theorem 10 being satisfied by $N=\tilde{S}$, in a particular case, we conclude that $N$ is the boundary of a Levi-flat $(2 n-2)$ variety (with negligible singularities) $\tilde{M}$ in $\mathbf{R} \times \mathbb{C}^{n}$.

Taking $\pi: \mathbb{C} \times \mathbb{C}^{n} \rightarrow \mathbb{C}^{n}$ to be the standard projection, we obtain the conclusion.

\subsection{Generalizations: elementary models and their gluing.}

3.5.1. The examples and the proofs of the theorems when $S$ is homeomorphic to a sphere (sections 3.4) suggest the following definitions.

3.5.2. Definitions. Let $T^{\prime}$ be a smooth, locally closed (i.e. closed in an open set), connected submanifold of $\mathbb{C}^{n}, n \geq 3$. We assume that $T^{\prime}$ has the following properties:

(i) $T^{\prime}$ is relatively compact, non necessarily compact, and of codimension 2 .

(ii) $T^{\prime}$ is nonminimal at every CR point.

(iii) $T^{\prime}$ does not contain complex manifold of dimension $(n-2)$.

(iv) $T^{\prime}$ has exactly 2 complex points which are flat and either special elliptic or special 1-hyperbolic. 
$(v)$ If $p \in T^{\prime}$ is special 1-hyperbolic, the singular orbit $\Sigma^{\prime}$ through $p$ is compact, $\Sigma^{\prime} \backslash p$ has two connected components $\sigma_{1}, \sigma_{2}$, whose closures are homeomorphic to spheres of dimension $2 n-3$.

(vi) If $p \in T^{\prime}$ is special 1-hyperbolic, in the neighborhood of $p$, with convenient coordinates, the equation of $T^{\prime}$, up to third order terms is

$$
z_{n}=\sum_{j=1}^{n-1}\left(z_{j} \bar{z}_{j}+\lambda_{j} \mathcal{R} e z_{j}^{2}\right) ; \lambda_{1}>1 ; 0 \leq \lambda_{j}<1 \text { for } j \neq 1
$$

or in real coordinates $x_{j}, y_{j}$ with $z_{j}=x_{j}+i y_{j}$,

$$
x_{n}=\left(\left(\lambda_{1}+1\right) x_{1}^{2}-\left(\lambda_{1}-1\right) y_{1}^{2}\right)+\sum_{j=2}^{n-1}\left(\left(1+\lambda_{j}\right) x_{j}^{2}+\left(1-\lambda_{j}\right) y_{j}^{2}\right)+O\left(|z|^{3}\right)
$$

(vii) the closures, in $T^{\prime}, T_{1}, T_{2}, T_{3}$ of the three connected components $T_{1}^{\prime}, T_{2}^{\prime}, T_{3}^{\prime}$ of $T^{\prime} \backslash \Sigma^{\prime}$ are submanifolds with (singular) boundary. Let $T^{\prime \prime}{ }_{j}$, $j=1,2,3$ be neighborhoods of the $T_{j}^{\prime}$ in $T^{\prime}$.

up- and down-1-hyperbolic points. Let $\tau$ be the $(2 n-2)$-submanifold with (singular) boundary contained into $T^{\prime}$ such that either $\bar{\sigma}_{1}\left(\right.$ resp. $\bar{\sigma}_{2}$ ) is the boundary of $\tau$ near $p$, or $\Sigma^{\prime}$ is the boundary of $\tau$ near $p$. In the first case, we say that $p$ is 1-up, (resp. 2-up), in the second that $p$ is down. If $T^{\prime}$ is contained in a small enough neighborhood of $\Sigma^{\prime}$ in $\mathbb{C}^{n}$, such a $T^{\prime}$ will be called a local elementary model, more precisely it defines a germ of elementary model around $\Sigma$.

The union $T$ of $T_{1}, T_{2}, T_{3}$ and of the germ of elementary model around the singular orbit at every special 1-hyperbolic point is called an elementary model. $T$ behaves as a locally closed submanifold still denoted $T$.

3.5.3. Examples of elementary models. We will say that $T$ is a elementary model of type:

(a) if it has: two elliptic points;

(b) if it has: one special elliptic point and one down-\{1\}-hyperbolic point;

$\left(c_{1}\right)$ if it has: one special elliptic point and one 1-up-\{1\}-hyperbolic point;

$\left(c_{2}\right)$ if it has: one special elliptic point and one 2-up-\{1\}-hyperbolic point;

$\left(d_{1}\right)$ if it has: two special 1-up-\{1\}-hyperbolic points;

$\left(d_{2}\right)$ if it has: two special 2-up-\{1\}-hyperbolic points;

(e) if it has: two special down-\{1\}-hyperbolic points;

Other configurations are easily imagined.

The prescribed boundary of a Levi-flat hypersurface of $\mathbb{C}^{n}$ in DTZ05] and [DTZ10], whose complex points are flat and elliptic, is an elementary model of type $(a)$.

3.5.4. Properties of elementary models. For instance, $T$ is 1-up and has one special elliptic point, we solve the boundary problem as in $S_{1}$ in the proof of Theorem 16 . 
Proposition 17. Let $T$ be a local elementary model. Then, $T$ carries a foliation $\mathcal{F}$ of class $C^{\infty}$ with 1-codimensional $\mathrm{CR}$ orbits as compact leaves.

Proof. From the definition at the end of section 3.5.2 and Proposition 6 ,

3.5.5.

Theorem 18. Let $T$ be the elementary model there exists an open neighborhood $T$ " in $T$ ' carrying a smooth function $\nu: T " \rightarrow \mathbb{R}$ whose level sets are the leaves of a smooth foliation.

Proof. By removing small connected open saturated neighborhoods of every special elliptic point, and of $\Sigma^{\prime}$, the singular orbit through every special 1hyperbolic point $p$, we obtain, from $S \backslash \Sigma^{\prime}$, three compact manifolds $S_{j}$ ", $j=1,2,3$, with boundary,

(a) $S_{1}$ and $S_{2}$ containing one special elliptic point $e$ or one special 1hyperbolic point with the foliations $\mathcal{F}_{1}, \mathcal{F}_{2}$, from Propositions 1 and 17 .

(b) $S_{3}$ " with the foliation $\mathcal{F}_{3}$ of codimension 1 given by its $\mathrm{CR}$ orbits whose first cohomology group with values in $\mathbf{R}$ is 0 , near $e$, or $p$. It is easy to show that this later foliation is transversely oriented.

From the Thurston's Stability Theorem (see section 3.4.2), $S_{3}$ " is homeomorphic to $\mathbf{S}^{2 n-3} \times[0,1]$, foliated as a product, with CR orbits being of the form $\mathbf{S}^{2 n-3} \times\{x\}$ for $x \in[0,1]$; hence smooth functions $\nu_{1}, \nu_{2}, \nu_{3}$, whose level sets are the leaves of the foliations $\mathcal{F}_{1}, \mathcal{F}_{2}, \mathcal{F}_{3}$ respectively, and using a partition of unity the desired function $\nu$ on $T$.

\section{6 .}

Theorem 19. Let $T$ be an elementary model. Then there exists a Levi-flat $(2 n-1)$-subvariety $\tilde{M} \subset \mathbb{C} \times \mathbb{C}^{n}$ with boundary $\tilde{T}$ (in the sense of currents) such that the natural projection $\pi: \mathbb{C} \times \mathbb{C}^{n} \rightarrow \mathbb{C}^{n}$ restricts to a bijection which is a CR diffeomorphism between $\tilde{T}$ and $T$ outside the complex points of $T$.

Proof. The submanifold $T$ being, locally, a boundary of a Levi-flat hypersurface, is orientable. We now set $\tilde{T}=N=\operatorname{gr} \nu=\{(\nu(z), z): z \in S\} \subset$ $E \cong \mathbf{R} \times \mathbb{C}^{n-1}$. Let $T_{s}$ be the union of the flat complex points of $T$.

$\lambda: T \rightarrow \tilde{T}(z \mapsto \nu((z), z))$ is bicontinuous; $\left.\lambda\right|_{T \backslash T_{s}}$ is a diffeomorphism; moreover $\lambda$ is a CR map. Choose an orientation on $T$. Then $N$ is an (oriented) CR subvariety with the negligible set of singularities $\tau=\lambda\left(T_{s}\right)$.

Using Remark 11, at every point of $T \backslash T_{s}, d_{x_{1}} \nu \neq 0$, we see that condition (H) (section 3.1.1) is satisfied at every point of $N \backslash \tau$.

Then all the assumptions of Theorem 10 being satisfied by $N=\tilde{T}$, in a particular case, we conclude that $N$ is the boundary of a Levi-flat $(2 n-2)$ variety (with negligible singularities) $\tilde{M}$ in $\mathbf{R} \times \mathbb{C}^{n}$.

Taking $\pi: \mathbb{C} \times \mathbb{C}^{n} \rightarrow \mathbb{C}^{n}$ to be the standard projection, we obtain the conclusion. 


\subsection{Gluing of elementary models.}

3.7.1. The gluing happens between two compatible elementary models along boundaries, for instance down and 1-up. Remark that the gluing can only be made at special 1-hyperbolic points. More precisely, it can be defined as follows.

The assumed properties of the submanifold $S$ in section 2 in $\mathbb{C}^{n}$ have a meaning in any complex analytic manifold $X$ of complex dimension $n \geq 3$, and are kept under any holomorphic isomorphism.

We will define a submanifold $S^{\prime}$ of $X$ obtained by gluying of elementary models by induction on the number $m$ of models. An elementary model $T$ in $X$ is the image of an elementary model $T_{0}$ in $\mathbb{C}^{n}$ by an analytic isomorphism of a neighborhood of $T_{0}$ in $\mathbb{C}^{n}$ into $X$.

3.7.2. Let $S^{\prime}$ be a closed smooth real submanifold of $X$ of dimension $2 n-2$ which is non minimal at every CR point. Assume that $S^{\prime}$ is obtained by gluing of $m$ elementary models.

a) $S^{\prime}$ has a finite number of flat complex points, some special elliptic and the others special 1-hyperbolic;

b) for every special 1-hyperbolic $p^{\prime}$, there exists a CR-isomorphism $h$ induced by a holomorphic isomorphism of the ambient space $\mathbb{C}^{n}$ from a neighborhood of $p$ in $T^{\prime}$ onto a neighborhood of $p^{\prime}$ in $S^{\prime}$.

c) for every CR-orbit $\Sigma_{p^{\prime}}$ whose closure contains a special 1-hyperbolic point $p^{\prime}$, there exists a CR-isomorphism $h$ induced by a holomorphic isomorphism of the ambient space $\mathbb{C}^{n}$ from a neighborhood of $\Sigma_{p}=\Sigma_{p}^{\prime} \backslash p$ in $T^{\prime}$ onto a neighborhood $V$ of $\Sigma_{p^{\prime}}$ in $S^{\prime}$.

Every special 1-hyperbolic point of $S^{\prime}$ which belongs to only one elementary model in $S^{\prime}$ will be called free.

We will define the gluing of one more elementary model to $S^{\prime}$.

3.7.3. Gluing an elementary model $T$ of type $\left(d_{1}\right)$ to a free down-1-hyperbolic point of $S^{\prime}$. Let $h_{1}$ be a CR-isomorphism from a neighborhood $V_{1}$ of $\bar{\sigma}_{1}^{\prime}$ induced by a holomorphic isomorphism of the ambient space $\mathbb{C}^{n}$ onto a neighborhood of $\sigma_{1}$ in $S^{\prime}$. Let $k_{1}$ be a CR-isomorphism from a neighborhood $T^{\prime \prime}{ }_{1}$ of $T_{1}^{\prime}$ into $X$ such that $k_{1} \mid V_{1}=h_{1}$.

\subsection{4.}

Theorem 20. The compact manifold or the manifold with singular boundary $S^{\prime}$, obtained by the gluing of a finite number of elementary models, is the boundary of a Levi-flat hypersurface of $X$ in the sense of currents.

Proof. From Theorem 19 and the definition of gluing. 
3.8. Examples of gluing. Denoting the gluing of the two models of type $\left(d_{1}\right)$ and $\left(d_{2}\right)$ to a free down-1-hyperbolic point of $S^{\prime}$ by: $\rightarrow\left(d_{1}\right)-\left(d_{2}\right)$, and the converse by: $\left(d_{1}\right)-\left(d_{2}\right) \rightarrow$, and, also, analogous configurations in the same way, we get:

torus: $(b) \rightarrow\left(d_{1}\right)-\left(d_{2}\right) \rightarrow(b)$; the Euler-Poincaré characteristic of a torus is $\chi\left(\mathbf{T}^{k}\right)=0: 2$ special elliptic and 2 special 1-hyperbolic points.

bitorus: $(b) \rightarrow\left(d_{1}\right)-\left(d_{2}\right) \rightarrow(e) \rightarrow\left(d_{1}\right)-\left(d_{2}\right) \rightarrow(b)$.

\section{CASE OF GRAPHS}

(see [DTZ09] for the case of elliptic points only, and dropping the property of the function solution to be Lipschitz).

4.1. We want to add the following hypothesis: $S$ is embedded into the boundary of a strictly pseudoconvex domain of $\mathbb{C}^{n}, n \geq 3$, and more precisely, let $(z, w)$ be the coordinates in $\mathbb{C}^{n-1} \times \mathbb{C}$, with $z=\left(z_{1}, \ldots, z_{n-1}\right), w=$ $u+i v=z_{n}$, let $\Omega$ be a strictly pseudoconvex domain of $\mathbb{C}^{n-1} \times \mathbb{R}_{u}$ (i.e. the second fundamental form of the boundary $b \Omega$ of $\Omega$ is everywhere positive definite); let $S$ be the graph $g r(g)$ of a smooth function $g: b \Omega \rightarrow \mathbb{R}_{v}$. notice that $b \Omega \times \mathbb{R}_{v}$ contains $S$ and is strictly pseudoconvex.

Assume that $S$ is a horned sphere (section 3.4), satisfying the hypotheses of Theorem 16. Denote by $p_{j}, j=i, \ldots, 4$ the complex points of $S$. Our aim is to prove

4.2 .

Theorem 21. Let $S$ be the graph of a smooth function $g: b \Omega \rightarrow \mathbb{R}_{v}$. Let $Q=$ $\left(q_{1}, \ldots, q_{4}\right) \in b \Omega$ be the projections of the complex points $P=\left(p_{1}, \ldots, p_{4}\right)$ of $S$, respectively. Then, there exists a continuous function $f: \bar{\Omega} \rightarrow \mathbb{R}_{v}$ which is smooth on $\bar{\Omega} \backslash Q$ and such that $f_{\mid b \Omega}=g$, and $M_{0}=\operatorname{graph}(f) \backslash S$ is a smooth Levi flat hypersurface of $\mathbb{C}^{n}$. Moreover, each complex leaf of $M_{0}$ is the graph of a holomorphic function $\phi: \Omega^{\prime} \rightarrow \mathbb{C}$ where $\Omega^{\prime} \subset \mathbb{C}^{n-1}$ is a domain with smooth boundary (that depends on the leaf) and $\phi$ is smooth on $\bar{\Omega}^{\prime}$.

The natural candidate to be the graph $M$ of $f$ is $\pi(\tilde{M})$ where $\tilde{M}$ and $\pi$ are as in Theorem [16. We prove that this is the case proceeding in several steps.

\subsection{Behaviour near $S$.}

4.3.1. Assume that $D$ is a strictly pseudoconvex domain and that $S \subset b D$. Recall ([HL75][Theorem 10.4]: Let D be a strictly pseudoconvex domain of $\mathbb{C}^{n}, n \geq 3$ with boundary $b D, \Sigma \subset b D$ be a compact connected maximally complex smooth $(2 d-1)$-submanifold with $d \geq 2$. Then, $\Sigma$ is the boundary of a uniquely determined relatively compact subset $V \subset \bar{D}$ such that $\bar{V} \backslash \Sigma$ is a complex analytic subset of $D$ with finitely many singularities of pure 
dimension $\leq d-1$, and near $\Sigma, \bar{V}$ is a d-dimensional complex manifold with boundary.

$V$ is said to be the solution of the boundary problem for $\Sigma$.

4.3 .2 .

Lemma 22 ([DTZ09]). Let $\Sigma_{1}, \Sigma_{2}$ be compact connected maximally complex $(2 d-1)$-submanifolds of $b D$. Let $V_{1}, V_{2}$ be the corresponding solutions of the boundary problem. If $d \geq 2,2 d \geq n+1$ and $\Sigma_{1} \cap \Sigma_{2}=\emptyset$, then $V_{1} \cap V_{2}=\emptyset$.

Let $\Sigma$ be a CR orbit of the foliation of $S \backslash P$. Then $\Sigma$ is a compact maximally complex $(2 n-3)$-dimensional real submanifold of $\mathbb{C}^{n}$ contained in $b D$. Let $V=V_{\Sigma}$ be the solution of the boundary problem corresponding to $\Sigma$. From Theorem [16, $V=\pi(\tilde{V})$, where $\tilde{V}=(\tilde{M} \backslash \tilde{S}) \cap\left(\mathbb{C}^{n} \times\{x\}\right)$ for suitable $x \in(0,1)$, the projection on the $x$-axis being finite, we can always assume that it lies into $(0,1)$. Moreover $\pi_{\mid \tilde{V}}$ is a biholomorphism $\tilde{V} \cong V$ and $M \backslash S \subset D$.

Let $\Sigma_{1}, \Sigma_{2}$ be two distinct orbits of the foliation of $S \backslash P$, and $\bar{V}_{1}, \bar{V}_{2}$ the corresponding leaves, then, from Lemma 22, $\bar{V}_{1} \cap \bar{V}_{2}=\emptyset$.

4.3.3. Assume that $S$ satisfies the full hypotheses of Theorem 21.

Set $m_{1}=\min _{S} g, m_{2}=\max _{S} g$ and $r \gg 0$ such that

$$
D=\Omega \times\left[m_{1}, m_{2}\right] \subset \subset \mathbf{B}(\mathbf{r}) \cap\left(\Omega \times i \mathbb{R}_{v}\right)
$$

where $\mathbf{B}(\mathbf{r})$ is the ball $\{|(z, w)|<r\}$.

\subsection{4.}

Lemma 23. Let $p \in S$ be a $C R$ point. Then, near $p, M$ is the graph of a function $\phi$ on a domain $U \subset \mathbb{C}_{z}^{n-1} \times \mathbb{R}_{u}$ which is smooth up to the boundary of $U$.

Proof. Near $p$, each CR orbit $\Sigma$ is smooth and can be represented as the graph of a CR function over a strictly pseudoconvex hypersurface and $V_{\Sigma}$ as the graph of the local holomorphic extension of this function. From Hopf lemma, $V$ is transversal to the strictly pseudoconvex hypersurface $d \Omega \times i \mathbb{R}_{v}$ near $p$. Hence the family of the $V_{\Sigma}$, near $p$, forms a smooth real hypersurface with boundary on $S$ that is the graph of a smooth function $\phi$ from a relative open neighborhood $U$ of $p$ on $\bar{\Omega}$ into $\mathbb{R}_{v}$. Finally, Lemma 22 garantees that this family does not intersect any other leaf $V$ from $M$.

Corollary 24. If $p \in S$ is a $C R$ point, each complex leaf $V$ of $M$, near $p$, is the graph of a holomorphic function on a domain $\Omega_{V} \subset \mathbb{C}_{z}^{n-1}$, which is smooth up to the boundary of $\Omega_{V}$.

\subsection{Solution as a graph of a continuous function.}


4.4.1. Recall results of Shcherbina Shc93 from:

(a) the Main Theorem:

Let $G$ be a bounded strictly convex domain in $\mathbb{C}_{z} \times \mathbb{R}_{u}(z \in \mathbb{C})$ and $\varphi: b G \rightarrow \mathbb{R}_{v}$ be a continuous function. Then the following properties hold, where $\Gamma=$ gr, and $\hat{\Gamma}(\varphi)$ means polynomial hull of $\Gamma(\varphi)$ :

$\left(a_{i}\right)$ the set $\hat{\Gamma}(\varphi) \backslash \Gamma(\varphi)$ is the union of a disjoint family of complex discs $\left\{D_{\alpha}\right\}$;

$\left(a_{i i}\right)$ for each $\alpha$, there is a simply connected domain $\Omega_{\alpha} \subset \mathbb{C}_{z}$ and a holomorphic function $w=f_{\alpha}$, defined on $\Omega_{\alpha}$, such that $D_{\alpha}$ is the graph of $f_{\alpha}$.

$\left(a_{i i i}\right)$ For each $f_{\alpha}$, there exists an extension $f_{\alpha}^{*} \in C\left(\bar{\Omega}_{\alpha}\right)$ and $b D_{\alpha}=$ $\left\{(z, w) \in b \Omega_{\alpha} \times \mathbb{C}_{w}: w=f_{\alpha}^{*}(z)\right\}$.

(b)

Lemma 25. Let $\left\{G_{n}\right\}_{n=0}^{\infty}, G_{n} \subset \mathbb{C}_{z} \times \mathbb{R}_{u}$, be a sequence of bounded strictly convex domains such that $G_{n} \rightarrow G_{0}$. Let $\left\{\varphi_{n}\right\}_{n=0}^{\infty}, \varphi_{n}: \partial G_{n} \rightarrow \mathbb{R}_{v}$ be a sequence of continuous functions such that $\Gamma\left(\varphi_{n}\right) \rightarrow \Gamma\left(\varphi_{0}\right)$ in the Hausdorff metric. Then, if $\Phi_{n}$ is the continuous function $: \bar{G}_{n} \rightarrow \mathbb{R}_{v}$ such that $\hat{\Gamma}(\varphi)=$ $\Gamma(\Phi)$, we have $\Gamma\left(\Phi_{n}\right) \rightarrow \Gamma\left(\Phi_{0}\right)$ in the Hausdorff metric.

(c)

Lemma 26. Let $\mathcal{U}$ be a smooth connected surface which is properly embedded into some convex domain $G \subset \mathbb{C}_{z} \times \mathbb{R}_{u}$. Suppose that near each point of this surface, it can be defined locally by the equation $u=u(z)$. Then the surface $\mathcal{U}$ can be represented globally as a graph of some function $u=U(z)$, defined on some domain $\Omega \subset \mathbb{C}_{z}$.

\subsection{2.}

Proposition 27. $M$ is the graph of a continuous function $f: \bar{\Omega} \rightarrow \mathbb{R}_{v}$.

Proof. We will intersect the graph $S$ with a convenient affine subspace of real dimension 4 to go back to the situation of Shcherbina.

Fix $a \in\left(\mathbb{C}_{z}^{n-1} \backslash 0\right)$ and, for a given point $(\zeta, \xi) \in \Omega$, with $\zeta \in \mathbb{C}_{z}^{n-1}$ and $\xi \in \mathbb{R}_{u}$, let $H_{(\zeta, \xi)} \subset \mathbb{C}_{z}^{n-1} \times\{\xi\}$ be the complex line through $(\zeta, \xi)$ in the direction $(a, 0)$. Set:

$L_{(\zeta, \xi)}=H_{(\zeta, \xi)}+\mathbb{R}_{u}(0,1), \quad \Omega_{(\zeta, \xi)}=L_{(\zeta, \xi)} \cap \Omega, \quad S_{(\zeta, \xi)}=\left(H_{(\zeta, \xi)}+\mathbb{C}_{w}(0,1)\right) \cap S$

Then $S_{(\zeta, \xi)}$ is contained in the strictly convex cylinder

$$
\left(H_{(\zeta, \xi)}+\mathbb{C}_{w}(0,1)\right) \cap\left(b \Omega \times i \mathbb{R}_{v}\right)
$$

and is the graph of $g_{\mid b \Omega_{(\zeta, \xi)}}$.

From $\left(a_{i i}\right)$, the polynomial hull of $S_{(\zeta, \xi)}$ is a continuous graph over $\bar{\Omega}_{(\zeta, \xi)}$. Consider $M=\pi(\tilde{M})$ and set

$$
M_{\zeta, \xi)}=\left(H_{(\zeta, \xi)}+\mathbb{C}_{w}(0,1)\right) \cap M .
$$


It follows that $M_{\zeta, \xi)}$ is contained in the polynomial hull $\hat{S}_{(\zeta, \xi)}$. From $\left(a_{i i i}\right)$, $\hat{S}_{(\zeta, \xi)}$ is a graph over $\bar{\Omega}_{(\zeta, \xi)}$ foliated by analytic discs, so $M_{\zeta, \xi)}$ is a graph over a subset $U$ of $\bar{\Omega}_{(\zeta, \xi)}$.

Every analytic disc $\Delta$ of $\hat{S}_{(\zeta, \xi)}$ had its boundary on $S_{(\zeta, \xi)}$. Since all the the complex points of $S$ are isolated, $b \Delta$ contains a CR point $p$ of $S$; from Lemma 23, near $p, M_{\zeta, \xi)}$ is a graph over $\bar{\Omega}_{(\zeta, \xi)}$. Near $p, \Delta$ is contained in $M_{\zeta, \xi)}$, then in a closed complex analytic leaf $V_{\Sigma}$ of $M$; so $\Delta \subset V_{\Sigma} \subset M$; but $\Delta \subset H_{(\zeta, \xi)}+\mathbb{C}_{w}(0,1)$; then: $\Delta \subset M_{\zeta, \xi)}$. Consequently, near $p, M_{\zeta, \xi)}=\hat{S}_{(\zeta, \xi)}$. It follows that $M$ is the graph of a function $f: \bar{\Omega} \rightarrow \mathbb{R}_{v}$.

One proves, using (b), that $f$ is continuous on $\Omega$, whence on $\bar{\Omega} \backslash Q$, by Lemma 23. Then continuity at every $q_{j}$ is proved using the Kontinuitätsatz on the domain of holomorphy $\Omega \times i \mathbb{R}_{v}$.

4.5. Regularity. The property: $M \backslash P=\left(p_{1}, \ldots, p_{4}\right)$ is a smooth manifold with boundary results from:

\subsection{1.}

Lemma 28. Let $U$ be a domain of $\mathbb{C}_{z}^{n-i} \times \mathbb{R}_{u}, n \geq 2, f: U \rightarrow \mathbb{R}_{v}$ a continuous function. Let $A \subset \operatorname{graph}(f)$ be a germ of complex analytic set of codimension 1. Then $A$ is a germ of complex manifold which is a graph of over $\mathbb{C}_{z}^{n-i}$.

Proof. Assume that $A$ is a germ at 0 . Let $g \in \mathcal{O}, h \neq 0$ such that $A=\{h=$ $0\}$. For $\varepsilon<<1$, let $\mathbf{D}_{\varepsilon}$ be the disc $\{z=0\} \cap\{|w|<\varepsilon\}$, then $A \cap \mathbf{D}_{\varepsilon}=\{0\}$, i.e. $A$ is $w$-regular.

Let $\pi: \mathbb{C}_{z, w}^{n} \rightarrow \mathbb{C}_{z}^{n-1}$ be the projection. The local structure theorem for analytic sets gives:

for some neighborhood $U$ of 0 in $\mathbb{C}_{z}^{n-1}$, there exists an analytic hypersurface $\Delta \subset U$ such that: $A_{\Delta}=A \cup\left((U \backslash \Delta) \times \mathbf{D}_{\varepsilon}\right)$ is a manifold;

$\pi / A_{\Delta} \rightarrow U \backslash \Delta$ is a $d(\in \mathbb{N})$-sheeted covering.

It is easy to show that the covering $\pi: A_{\Delta} \rightarrow U \backslash \Delta$ is trivial.

Then we may define $d$ holomorphic functioons $\tau_{1}, \ldots, \tau_{d}: U \backslash \Delta \rightarrow \mathbb{C}$ such that $A_{\Delta}$ is the union of the graphs of the $\tau_{j}$. By the Riemann extension theorem, the functions $\tau_{j}$ extend as holomorphic functions $\tau_{j} \in \mathcal{O}(U)$. Suppose that $\tau_{j} \neq \tau_{k}$, for $j \neq k$, then for some disc $\mathbf{D} \subset U$ centered at 0 , we have $\tau_{j}\left|\mathbf{D} \neq \tau_{k}\right| \mathbf{D}$, then $\left.\left(\tau_{j}-\tau_{k}\right)\right|_{\mathbf{D}}$ vanishes only at 0 . But, from the hypothesis, in restriction to $\mathbf{D},\left.\left\{\operatorname{Re}\left(\tau_{j}-\tau_{k}\right)=0\right\} \subset\left\{\tau_{j}-\tau_{k}=0\right\}\right|_{\mathbf{D}}=\{0\}$, impossible.

\section{6 .}

Proof of the Theorem [21. Consider the foliation of $S \backslash P$ given by the level sets of the smooth function $\nu: S \rightarrow[0,1]$ (sections 2.3 and 2.7) and set $L_{t}=\{\nu=t\}$ for $t \in(0.1)$. Let $V_{t} \subset \bar{\Omega} \times i \mathbb{R}_{v} \subset \mathbb{C}^{n}$ be the complex leaf of $M$ bounded by $L_{t}$. 
By Proposition 27, $M$ is the graph of a continuous function over $\Omega$, and, by Lemma 28, each leaf $V_{t}$ is a complex smooth hypersurface and $\left.\pi\right|_{V_{t}}$ is a submersion.

$\rightarrow$

Since $\Omega$ is strictly convex, as in Shcherbina (see 4.4.1, c)), $\pi_{\mid V_{t}}$ is 1-1, then, by Corollary 24, $\pi$ sends $V_{t}$ onto a domain $\Omega_{t} \subset \mathbb{C}_{z}^{n-1}$ with smooth boundary. Let

$$
\begin{aligned}
& \pi_{u}:\left(\mathbb{C}_{z}^{n-1} \times \mathbb{R}_{u}\right) \times i \mathbb{R}_{v} \rightarrow \mathbb{R}_{u} \\
& \pi_{v}:\left(\mathbb{C}_{z}^{n-1} \times \mathbb{R}_{u}\right) \times i \mathbb{R}_{v} \rightarrow \mathbb{R}_{v}
\end{aligned}
$$

then $\pi_{u \mid L_{t}}=a_{t} \cdot \pi_{\mid L_{t}}$ and $\pi_{v \mid L_{t}}=b_{t} \cdot \pi_{\mid L_{t}}$ where $a_{t}, b_{t}$ are smooth functions on $b \Omega_{t}$. Moreover $b \Omega_{t}, a_{t}, b_{t}$ depend smoothly on $t$.

If $\left(z_{t}, w_{t}\right) \in M$, then $w_{t}$ varies on $V_{t}$, so $w_{t}$ is the holomorphic extension of $a_{t}+i b_{t}$ to $\Omega_{t}$. In particular $u_{t}$ and $v_{t}$ are smooth in $(z, t)$, from the Bochner-Martinelli formula.

$\frac{\partial u_{t}}{\partial t}$ is harmonic on $\Omega_{t}$ for each $t$ and has a smooth extension on $b \Omega_{t}$.

From Lemma 23 and Corollary 24, $\frac{\partial u_{t}}{\partial t}$ does not vanish on $b \Omega_{t}$. Since the CR orbits $L_{t}$ are connected from Proposition [14, $b \Omega_{t}$ is also connected, hence $\frac{\partial u_{t}}{\partial t}$ has constant sign on $b \Omega_{t}$. Then, by the maximum principle, also $\frac{\partial u_{t}}{\partial t}$ on $\Omega_{t}$ and, in particular does not vanish. This implies that $M \backslash S$ is the graph of a smooth function over $\Omega$ which smoothly extends to $\bar{\Omega} \backslash Q$.

From Proposition 27, $M$ is the graph of a continuous function over $\bar{\Omega}$.

\subsection{Elementary smooth models.}

4.7.1. Definition. An elementary smooth model in $\mathbb{C}^{n}$ is an elementary model in the sense of section 3.5 .2 and satisfying the further condition which makes sense from Theorem 21,

$(\mathrm{G})$ Let $(z, w)$ be the coordinates in $\mathbb{C}^{n-1} \times \mathbb{C}$, with $z=\left(z_{1}, \ldots, z_{n-1}\right), w=$ $u+i v=z_{n}$, let $\Omega$ be a strictly pseudoconvex domain of $\mathbb{C}^{n-1} \times \mathbb{R}_{u}$; assume that $T^{\prime}$ is the graph of a smooth function $g: b \Omega \rightarrow \mathbb{R}_{v}$.

Theorem 29. Let $T$ be an elementary smooth model. Then, there exists a continuous function $f: \bar{\Omega} \rightarrow \mathbb{R}_{v}$ which is smooth on $\bar{\Omega} \backslash Q$ and such that $f_{\mid b \Omega}=g$, and $M_{0}=\operatorname{graph}(f) \backslash S$ is a smooth Levi flat hypersurface of $\mathbb{C}^{n}$; in particular, $S$ is the boundary of the hypersurface $M=\operatorname{graph}(f)$

Proof. similar to the proof of Theorem 21.

4.7.3. Gluing of elementary smooth models. In an open set of $\mathbb{C}^{n}$, a coordinate system $(z, w)$ of $\mathbb{C}_{z}^{n-1} \times \mathbb{R}_{u}$ defines an $(n-1,1)$-frame.

To define the gluing of elementary models (section 3.7) we considered a CR-isomorphism from an open set of $\mathbb{C}^{n}$ induced by a holomorphic isomorphism of the ambient space $\mathbb{C}^{n}$ onto a an open set of $\mathbb{C}^{n}$. To define the 
gluing of elementary smooth models, we have to consider a holomorphic isomorphism of the ambient space $\mathbb{C}^{n}$ onto an open set of $\mathbb{C}^{n}$ sending an $(n-1,1)$-frame of $\mathbb{C}_{z}^{n-1} \times \mathbb{R}_{u}$ onto an $(n-1,1)$-frame of $\mathbb{C}_{z^{\prime}}^{n-1} \times \mathbb{R}_{u^{\prime}}$.

As in section 3.7.1, we will define a submanifold $S^{\prime}$ of $X$ obtained by gluing of elementary smooth models by induction on the number $m$ of models. An elementary smooth model $T$ in $X$ is the image of an elementary smooth model $T_{0}$ of $\mathbb{C}^{n}$ by an analytic isomorphism of a neighborhood of $T_{0}$ in $\mathbb{C}^{n}$ into $X$.

Gluing an elementary smooth model $T$ of type $\left(d_{1}\right)$ to a free down-1-hyperbolic point of $S^{\prime}$.

Every elementary smooth model is contained in a cylinder $b \Omega \times \mathbb{R}_{v}$ determined by $\Omega$ and an $(n-1,1)$-frame. Two sets $\Omega$ are compatible if either they coincide or one is part of the other.

The announced gluing is defined in the following way: there exists a CRisomorphism $h_{1}$ from a neighborhood $V_{1}$ of $\bar{\sigma}_{1}^{\prime}$ induced by a holomorphic isomorphism of the ambient space $\mathbb{C}^{n}$ onto a neighborhood of $\sigma_{1}$ in $S^{\prime}$. Let $k_{1}$ be a CR-isomorphism from a neighborhood $T{ }^{\prime}{ }_{1}$ of $T_{1}^{\prime}$ into $X$ such that $k_{1} \mid V_{1}=h_{1}$, and there exists a common $(n-1,1)$-frame on which the corresponding sets $\Omega$ are compatible. The existence of such a situation is possible as the example of the horned (almost everywhere) smooth sphere shows (Theorem 21.).

Remark that the gluing implies that the obtained submanifold $S^{\prime}$ is $C^{0}$ and smooth except at the complex points.

Other gluing are obtained in a similar way. Hence:

Theorem 30. The manifold $S^{\prime}$ obtained by gluing of elementary smooth models is of class $C^{0}$, and smooth except at the complex points.

Corollary 31. The manifold $S^{\prime}$ is the boundary of manifold $M$ of class $C^{\infty}$ whose interior is a Levi-flat smooth hypersurface.

\section{REFERENCES}

[Bis65] Errett Bishop, Differentiable manifolds in complex Euclidean space, Duke Math. J. 32 (1965), 1-21. MR 0200476 (34 \#369)

[BK91] Eric Bedford and Wilhelm Klingenberg, On the envelope of holomorphy of a 2-sphere in $\mathbf{C}^{2}$, J. Amer. Math. Soc. 4 (1991), no. 3, 623-646. MR 1094437 (92j:32034)

[CS51] Shiing-shen Chern and E. Spanier, A theorem on orientable surfaces in fourdimensional space, Comment. Math. Helv. 25 (1951), 205-209. MR 0044883 $(13,492 \mathrm{~d})$

[Dol08] Pierre Dolbeault, On Levi-flat hypersurfaces with given boundary in $\mathbb{C}^{n}$, Sci. China Ser. A 51 (2008), no. 4, 541-552. MR 2395404 (2009c:32067)

[Dol11] P. Dolbeault, Complex plateau problem: old and new results and prospects, Arxiv preprint arXiv:1105.4417 (2011).

[DTZ05] Pierre Dolbeault, Giuseppe Tomassini, and Dmitri Zaitsev, On boundaries of Levi-flat hypersurfaces in $\mathbb{C}^{n}$, C. R. Math. Acad. Sci. Paris 341 (2005), no. 6, 343-348. MR 2169149 (2006e:32048) 
[DTZ09] P. Dolbeault, G. Tomassini, and D. Zaitsev, Boundary problem for Levi flat graphs, Arxiv preprint arXiv:0912.1332 (2009).

[DTZ10] Pierre Dolbeault, Giuseppe Tomassini, and Dmitri Zaitsev, On Levi-flat hypersurfaces with prescribed boundary, Pure Appl. Math. Q. 6 (2010), no. 3, Special Issue: In honor of Joseph J. Kohn. Part 1, 725-753. MR 2677311 (2011f:32074)

[HL75] F. Reese Harvey and H. Blaine Lawson, Jr., On boundaries of complex analytic varieties. I, Ann. of Math. (2) 102 (1975), no. 2, 223-290. MR 0425173 (54 \#13130)

[Lai72] Hon Fei Lai, Characteristic classes of real manifolds immersed in complex manifolds, Trans. Amer. Math. Soc. 172 (1972), 1-33. MR 0314066 (47 \#2618)

[Lai74] On the topology of the even-dimensional complex quadrics, Proc. Amer. Math. Soc. 46 (1974), 419-425. MR 0358846 (50 \#11305)

[Pol08] P. Polo, Grassmanniennes orientées réelles, Personnal communication, 1 fév. 2008.

[Shc93] N. V. Shcherbina, On the polynomial hull of a graph, Indiana Univ. Math. J. 42 (1993), no. 2, 477-503. MR 1237056 (95e:32017)

[Ste99] Norman Steenrod, The topology of fibre bundles, Princeton Landmarks in Mathematics, Princeton University Press, Princeton, NJ, 1999, Reprint of the 1957 edition, Princeton Paperbacks. MR 1688579 (2000a:55001)

Institut de Mathématiques de Jussieu, UPMC, 4, Place Jussieu 75005 Paris

E-mail address: pierre.dolbeault@upmc.fr 